\title{
Quaternary thermoelectric materials: synthesis, microstructure and thermoelectric properties of the $(\mathrm{Bi}, \mathrm{Sb})_{2}(\mathrm{Te}, \mathrm{Se})_{3}$ alloys
}

Xin Guo ${ }^{\mathrm{a}, *}$, Jieming Qin ${ }^{\mathrm{a}}$, Xiaopeng Jia ${ }^{\mathrm{b}}$, Hongan Ma ${ }^{\mathrm{b}}$, Hongsheng $\mathrm{Jia}^{\mathrm{c}}$

${ }^{a}$ Institute of Materials Science and Engineering, Changchun University of Science and Technology, Changchun 130022, China

${ }^{\mathrm{b}}$ State Key Laboratory of Superhard Materials, Jilin University, Changchun 130012, China

${ }^{\mathrm{c}}$ Key Laboratory of Functional Materials Physics and Chemistry of the Ministry of Education, Jilin Normal University, Siping 136000, China

\begin{abstract}
High-quality quaternary $\mathrm{Bi}_{0.5} \mathrm{Sb}_{1.5} \mathrm{Te}_{3-\mathrm{x}} \mathrm{Se}_{\mathrm{x}}$ (BSTS) alloys with $\mathrm{x}=0.3,0.5$ and 0.75 are successfully synthesized in 25 min by high pressure and high temperature (HPHT) method. Due to the effects of synthesis pressure and Se doping, the texture and microstructure exhibit abundant distorted layers and lattice defects. The unique triangular nanoplates can be obtained in the microstructures by HPHT method without any substrates, suggesting that $\mathrm{Bi}_{0.5} \mathrm{Sb}_{1.5} \mathrm{Te}_{3-\mathrm{x}} \mathrm{Se}_{\mathrm{x}}$ has a nature of the epitaxial growth. The thermoelectric transport properties are characterized in detail, revealing an evident correlation with the amount of Se doping. As a result, a maximum ZT value of 0.95 is achieved at 503K from the as-prepared $\mathrm{Bi}_{0.5} \mathrm{Sb}_{1.5} \mathrm{Te}_{2.7} \mathrm{Se}_{0.3}$ alloys. Additionally, Raman spectra are conducted and then indicate that Se doping contents have an important influence on the lattice vibrations and lattice structures of quaternary $(\mathrm{Bi}, \mathrm{Sb})_{2}(\mathrm{Te}, \mathrm{Se})_{3}$ alloys.
\end{abstract}

Keywords: Thermoelectric materials; High-pressure; $(\mathrm{Bi}, \mathrm{Sb})_{2}(\mathrm{Te}, \mathrm{Se})_{3}$; Microstructure; Raman spectra

\section{Introduction}

Thermoelectric materials, because of the important applications in the refrigeration and power generator, have attracted extensive interest in terms of relieving the energy crisis and environmental pollution [1]. However, the large-scale applications are restricted for now due to the low efficiency of thermoelectric conversion, which is evaluated by the dimensionless figure of merit (ZT) defined by $\mathrm{ZT}=\mathrm{TS}^{2} / \rho \kappa$, where $\mathrm{T}$ is the kelvin temperature, and the interdependent thermoelectric parameters $\mathrm{S}, \rho$

\footnotetext{
* Corresponding author. E-mail: guoyml@126.com (X. Guo)
} 
and $\kappa$ are the Seebeck coefficient, electrical resistivity and thermal conductivity, respectively $[2,3]$. To date, most of the researches for the improvement of thermoelectric properties almost revolve around the two-dimensional structures/nanostructures design, composition optimization, technical innovation, etc., especially in the study of $\mathrm{Bi}_{2} \mathrm{Te}_{3}$-based materials.

$\mathrm{Bi}_{2} \mathrm{Te}_{3}$-based materials as the most promising candidates exhibit noticeable improvement of the $\mathrm{ZT}$ values in recent years, which is attributed to the optimization of structure and chemical composition [4]. As is well known, the $\mathrm{Sb}$ and $\mathrm{Se}$ elements as effective dopants can generally obtain high-quality p-type $(\mathrm{Bi}, \mathrm{Sb})_{2} \mathrm{Te}_{3}$ and $\mathrm{n}$-type $\mathrm{Bi}_{2}(\mathrm{Te}, \mathrm{Se})_{3}[5-7]$. However, the synthesis, microstructure and thermoelectric performance of the quaternary $(\mathrm{Bi}, \mathrm{Sb})_{2}(\mathrm{Te}, \mathrm{Se})_{3}$ are less reported comparing with the above ternary $\mathrm{Bi}_{2} \mathrm{Te}_{3}$-based alloys. To analyze the causes, we summarize the following points: difficult synthesis with conventional methods, complex technology and froward thermoelectric performance. With the development of high-pressure techniques, pressure as a well-developed technique is widely used in the preparation of thermoelectric materials, such as hot press [8], spark plasma sintering [9], extrusion methods [10] and high-pressure synthesis [11], which exhibit respective advantages in terms of optimizing thermoelectric performances. In addition, our previous works indicate that high pressure and high temperature (HPHT) method for thermoelectric materials have some unique superiorities including rapid preparation, low-cost, one-step, pure phase obtained readily, performance optimization, microstructure modulation, etc. [12-14]. Therefore, in allusion to above difficult in quaternary $(\mathrm{Bi}, \mathrm{Sb})_{2}(\mathrm{Te}, \mathrm{Se})_{3}$ alloys, the HPHT mothed is employed to conduct the preparation and investigation of quaternary $(\mathrm{Bi}, \mathrm{Sb})_{2}(\mathrm{Te}, \mathrm{Se})_{3}$ alloys in this work.

$\mathrm{Bi}_{0.5} \mathrm{Sb}_{1.5} \mathrm{Te}_{3-\mathrm{x}} \mathrm{Se}_{\mathrm{x}}$ alloys with various Se contents are successfully synthesized in $25 \mathrm{~min}$ via HPHT mothed. The unique triangular nanoplates and lattice defects are observed readily in the mircostructure. Raman spectra suggest that Se doping can significantly impact on the lattice vibrations and lattice structures of as-prepared $\mathrm{Bi}_{0.5} \mathrm{Sb}_{1.5} \mathrm{Te}_{3-\mathrm{x}} \mathrm{Se}_{\mathrm{x}}$ alloys. With the increase of Se contents, the thermoelectric transport properties reveal an evident diffenerce. Ultimately, a maximum ZT of 0.95 at $503 \mathrm{~K}$ is achieved from as-prepared p-type $\mathrm{Bi}_{0.5} \mathrm{Sb}_{1.5} \mathrm{Te}_{0.7} \mathrm{Se}_{0.3}$ alloy, which is significantly higher than the maximum ZT value of p-type $(\mathrm{Bi}, \mathrm{Sb})_{2}(\mathrm{Te}, \mathrm{Se})_{3}$ alloys obtained by theoretical calculation [15], and is very close to the maximum $\mathrm{ZT}$ value of the state-of-the-art $(\mathrm{Bi}, \mathrm{Sb})_{2}(\mathrm{Te}, \mathrm{Se})_{3}$ alloys [16].

\section{Experimental section}




\subsection{Sample preparation}

Stoichiometric amounts of $\mathrm{Bi}, \mathrm{Sb}, \mathrm{Te}, \mathrm{Se}$ elemental powders with $99.999 \%$ purity were weighed and mixed according to the nominal composition of $\mathrm{Bi}_{0.5} \mathrm{Sb}_{1.5} \mathrm{Te}_{3-\mathrm{x}} \mathrm{Se}_{\mathrm{x}}$ with $\mathrm{x}=0.3$ (BSTS-03), 0.5 (BSTS-05) and 0.75 (BSTS-75) in a glove box under a nitrogen gas atmosphere. The mixtures were prepared to be a $\varphi 10.5 \times 11 \mathrm{~mm}$ cylinder by cold compression, and then was assembled into a sample chamber of $23 \mathrm{~mm}$ with a graphite crucible as a heating unit, following by preparation at $900 \mathrm{~K}$ and under the pressure of $2.4 \mathrm{GPa}$ for $25 \mathrm{~min}$ via a cubic anvil high-pressure apparatus (SPD $6 \times 1200$ ). The pressure was calibrated by the change in resistance of standard substances. The synthesis temperature was measured by a Pt RH/Pt Rh6 thermocouple placing near the sample.

\subsection{Characterization}

The phase structures of the as-prepared samples were identified by X-ray diffraction (XRD, D/MAX-RA) with $\mathrm{Cu}-\mathrm{K}_{a}$ radiation. The morphologies were observed by field-emission scanning electron microscopy (FESEM, Magellan-400 FEI microscope). An energy dispersive spectrometer (EDS) system in FESEM equipment was employed for measuring the atomic ratio of the composition. The microstructures were obtained by a high-resolution transmission electron microscope (HRTEM, JEOL JEM-2200FS). Raman spectroscopy as a useful tool was employed to investigate the lattice vibrational modes of as-prepared samples. The Hall coefficient $\left(\mathrm{R}_{\mathrm{H}}\right)$, carrier concentration $\left(\mathrm{n}_{\mathrm{p}}\right)$ and carrier mobility $(\mu)$ were measured at room temperature by a HMS-7707 Hall measurement system (LakeShore). The electrical properties ( $\mathrm{S}$ and $\rho$ ) were measured simultaneously using a ZEM-3 apparatus (ULVAC-RIKO, Inc.). The thermal conductivity was calculated by the formula of $\kappa=C_{p} \lambda D$, where $C_{p}, \lambda$ and $D$ are the specific heat, thermal diffusivity and relative density, respectively. The $C_{p}$ measurement by DSC conversion was obtained using a Linseis STA PT-1750 equipment with the sapphire revision, the $\lambda$ was measured based on a laser flash technique (Netzsch LFA-427), the D was obtained by the Archimedes method. To consider the anisotropy of $\mathrm{Bi}_{2} \mathrm{Te}_{3}$-based alloys, the parameters above were measured along the cross-plane (i.e. $11 \mathrm{~mm}$ direction). In addition, the errors in $\mathrm{S}, \rho$ and $\kappa$ had been retested that did not exceed $\pm 3- \pm 5 \%$.

\section{Results and discussion}

\subsection{Phase characterization}

Fig. 1(a) shows the XRD patterns of the as-prepared BSTS-03, BSTS-05, BSTS-75 and the standard 
$\mathrm{Bi}_{0.5} \mathrm{Sb}_{1.5} \mathrm{Te}_{3}$ data card (PDF\#49-1713). It can be observed that the characteristic peaks of the as-prepared samples can be indexed to the standard diffraction peaks (PDF\#49-1713), implying that the as-prepared samples have a rhombohedral lattice structure with the space group of R-3m. In addition, note that the characteristic peaks are shifted to larger $2 \theta$ angles with increasing Se contents, the most obvious shifts for the (015) peaks have been shown in the inset of Fig. 1(a), indicating that the local Te atoms in sublattices have been replaced by Se atoms with small atomic (ionic) radius. For further confirmation, the lattice parameters $(a, c)$ are calculated quantificationally by the peak positions of the (015) and (1010) planes. As shown in Fig. 1(b), the lattice parameters (a, c) exhibit a decrease with Se contents from 0.3 to 0.75 . The results suggest that the Se atoms have entered into the lattices to substitute for local Te atoms sites, successfully synthesizing the BSTS alloys by HPHT. Moreover, to check further orientation degrees of the (001) planes, the Lotgering method is employed, and then is calculated to be $0.15,0.13$ and 0.14 for the as-prepared BSTS-03 BSTS-05 and BSTS-75, respectively, which means that there is not obvious growth orientation due to multiple components and appropriate pressure.

\subsection{FESEM and HRTEM micrographs}

FESEM images in Fig. 2 exhibit the universal morphologies for the as-prepared BSTS alloys by HPHT, which have the typical lamellar structures of $\mathrm{Bi}_{2} \mathrm{Te}_{3}$-based alloys (the low magnification FESEM images in Fig. S1). The abundant lamellar groups can be observed readily, which are constituted by two-dimensional layer structures. Interestingly, the lamellar groups present distorted features and cleavage characteristics due to the effect of synthesis pressure on the $\mathrm{Te}^{(1)}-\mathrm{Te}^{(1)}$ bonding with weaker van der Waals force, moreover, the slippage and extrusion between the layers constitute widespread and irregular interspaces. As we all know, the textures with the above features are generally regarded as a positive effect for hindering the phonons and electrons transport, effectively scattering vast phonons in comparison with electrons to reduce lattice thermal conductivity. In addition, the EDS measurements for as-prepared BSTS-03, BSTS-05 and BSTS-75 are conducted in order to verify the atomic ratios of compositions. The results indicate that the atomic ratios for each of the chemical elements are approximate to the nominal composition, as shown in Fig. S2.

The TEM image of the as-prepared BSTS-03 alloys in Fig. 3(a) is characterized, revealing a lot of the nanoplates with regular edge, especially the triangular nanoplates, which are dispersed around the major crystals, and are constituted by abundant point defects (the volatilization of Te and Se atoms or 
the inherent Te vacancies) that can be observed in the inset of Fig.3(a). In addition, the triangular nanoplates are also obtained in other $\mathrm{Bi}_{2} \mathrm{Te}_{3}$-based alloys prepared by long-playing grind via high-energy ball-milling $[3,17]$. This means that the BSTS compounds have a nature of the epitaxial growth according to two-dimensional triangular lattice of the quintuple layers (QL) of the tetradymite without any substrates.

As shown in Fig. 3(b), the HRTEM image of the as-prepared BSTS-03 exhibits copious lattice orientations, and thus the irregular grain boundaries can be observed clearly. This means that the as-prepared samples by HPHT present a disorderly growth pattern and belong to the polycrystalline with a wide size distribution. Besides, the vast lattice defects originating from synthesis pressure and Se doping are revealed in Fig. 3(c) including the dislocations and lattice distortions. In order to observe the defects, the inverse fast fourier transform (IFFT) image corresponding to Fig. 3(c) is employed that can readily identify abundant dislocations and lattice distortions in white regions of Fig. 3(d). Generally, the observed microstructures above are responsible for a reduction in the phonon mean free paths, effectively scattering phonons and then realizing a decrease of thermal conductivity.

\subsection{Raman spectroscopy and lattice vibrations}

In order to investigate the effect of Se doping content on the lattice vibrations and lattice structure of as-prepared quaternary BSTS alloys, Raman spectra are excited at room temperature with the wavelength of $532 \mathrm{~nm}$ laser, and recorded in $50-200 \mathrm{~cm}^{-1}$, which are reproduced using Lorentzian line shape function fitting as shown in Fig. 4(a). Three Raman active modes can be clearly indexed assigning to $\mathrm{A}_{\mathrm{g}}{ }^{1}, \mathrm{E}_{\mathrm{g}}{ }^{2}$ and $\mathrm{Ag}_{\mathrm{g}}{ }^{2}$ vibration modes [18,19], which are the in-plane and cross-plane vibration modes for the E and A modes, respectively. It is noteworthy that the Raman shift (frequency) and relative intensity of $\mathrm{A}_{\mathrm{g}}{ }^{1}, \mathrm{E}_{\mathrm{g}}{ }^{2}$ and $\mathrm{A}_{\mathrm{g}}{ }^{2}$ vibration modes exhibit significant variations with increasing $\mathrm{Se}$ doping content in Fig. 4(b) and (c). As is well known, the $\mathrm{A}_{2} \mathrm{~B}_{3}(\mathrm{~A}=\mathrm{Bi}, \mathrm{Sb}$ and $\mathrm{B}=\mathrm{Te}, \mathrm{Se})$ has a rhombohedral crystal structure, and consists of the quintuple layers that are alternate arrangements of five atomic layers, $\mathrm{B}^{(1)}-\mathrm{A}-\mathrm{B}^{(2)}-\mathrm{A}-\mathrm{B}^{(1)}$, in which the $\mathrm{A}-\mathrm{B}^{(2)}$ is bonded by a pure covalent nature, and the A-B ${ }^{(1)}$ exhibits the chemical bonding with the ionic and covalent natures [20]. For the $\mathrm{Bi}_{0.5} \mathrm{Sb}_{1.5} \mathrm{Te}_{3-\mathrm{x}} \mathrm{Se}_{\mathrm{x}}$ compounds, the Se atoms, being smaller and more electronegative than Te atoms, preferentially replace $\mathrm{Te}$ atoms at $\mathrm{Te}^{(2)}$ sites, followed by random replacements of $\mathrm{Te}$ atoms at $\mathrm{Te}^{(1)}$ sites. Hence, the substantial enhancement in Raman shifts of $\mathrm{E}_{\mathrm{g}}{ }^{2}$ and $\mathrm{A}_{\mathrm{g}}{ }^{2}$ modes from the as-prepared BSTS-05 to BSTS-75 may be attributed to the $\mathrm{Te}^{(1)}$ sites substituted by Se atoms due to the similar saturated 
$\mathrm{Te}^{(2)} / \mathrm{Se}^{(2)}$ sites [21]. As a result, the crystalline structures and lattice dynamics of as-prepared BSTS will be changed significantly with the $\mathrm{Se} / \mathrm{Te}$ substituents, emerging strong atomic vibrations, Raman shift and relative intensity. Additionally, the existence of Sb atoms in BSTS may further strengthen the above variations in comparison with those of $\mathrm{Bi}_{2}(\mathrm{Te}, \mathrm{Se})_{3}$.

\subsection{Thermoelectric transport properties}

Fig. 5 shows the temperature dependences of the electrical resisitivity, Seebeck coefficient, thermal conductivity and ZT values of the as-prepared BSTS-03, BSTS-05 and BSTS-75 alloys. As shown in Fig. 5(a), the positive temperature dependence of electrical resisitivity exhibits a metal-like conduction or a degenerate semiconductor behavior. It can be seen that the electrical resisitivity seems to indicate two conduction mechanisms for the BSTS alloys with different Se doping contents. Note that the resisitivity is well described by a power law $\rho \sim \mathrm{T}^{3 / 2}$ for the BSTS-03 and BSTS-05. It is well known that the carrier mobility $(\mu)$ can be indicated by $\mu \sim T^{-3 / 2}$ for the temperature dependence, when the acoustic phonon scattering is the major mechanism in crystals [22]. Based on the formula of $\rho=1 / n e \mu$, it can be concluded that the carrier concentration (n) with temperature has a weak effect on the electrical resistivity of the BSTS-03 and BSTS-05 alloys. In contrast, the electrical resistivity of the as-prepared BSTS-75 exhibits a carrier transport behavior with temperature rise, which present a mild and even declining tendency in $600-660 \mathrm{~K}$ due to abundant carriers excited by high temperature. Due to high pressure and Se doping, the formative plastic deformation inside structure introduce high-density lattice defects and distortion, just like hot deformation (HD) and ball milling (BM), generating the donor-like effect to increase anion vacancies $\left(\mathrm{Te}^{2-}\right.$ and $\left.\mathrm{Se}^{2-}\right)$ [23-25], thus leading to a reduction in hole concentration of $\mathrm{Bi}_{0.5} \mathrm{Sb}_{1.5}(\mathrm{Te}, \mathrm{Se})_{3}$. For as-prepared BSTS-75, which depend on a carrier transport behavior, therefore exhibits an enhancement of resistivity comparing with that of as-prepared BSTS-03 and BSTS-05. In Fig. 5(b), the Seebeck coefficient exhibits a positive value, indicating that the quaternary BSTS alloys, when Se doping contents are in 0.3-0.75 mol., have a typical p-type semiconductor behavior. The Seebeck coefficient with temperature presents a parabola-like action, especially in that of BSTS-75, which is principally attributed to the effect of temperature on the carrier concentration, as previously mentioned in the resistivity. In addition, the Seebeck coefficient of as-prepared BSTS-75 is higher than that of others, which is mainly associated with two factors: (1) As mentioned above, the donor-like effect, which is induced by deformation generated by high pressure and Se doping, will decrease the carrier concentration to improve the Seebeck coefficient. In order to 
further ensure a reduction in carrier concentration, the Hall datum including Hall coefficient $\left(\mathrm{R}_{\mathrm{H}}\right)$, carrier concentration $\left(\mathrm{n}_{\mathrm{p}}\right)$ and carrier mobility $(\mu)$ have been shown in Table S1, and exhibit consistent results. (2) On the basis of the Vegard's law, the increased band gap with an amount of Se doping will impede abundant carriers across the wide band gap of the BSTS-75, leading to an increase of the Seebeck coefficient. In Fig. 5(c), the thermal conductivity of as-prepared BSTS alloys first reveals a reduction and then a rise with temperature. It can be easily understood that the scattering of electrons and phonons will notablely increase due to drastic lattice vibrations induced by thermal excitation at low temperture range. On the other hand, the donor-like effect and elevated temperature will give rise to an enhancement of the minority carriers $\left(\mathrm{V}_{\mathrm{Te}}{ }^{2-}\right.$ and $\left.\mathrm{V}_{\mathrm{Se}}{ }^{2-}\right)$, generating the competition effect between the charge and hole to increase the electron-hole coupling, and thus leading to a rise in the detrimental bipolar thermal conduction at high temperature $[25,26]$, especially the as-prepared BSTS- 75 with the carrier transport behavior. In addition, the lattice thermal conductivity is also calculated and plotted in Fig. S3 to further understand the effect of different transport mechanisms. The dimensionless ZT values of as-prepared samples are shown as functions of Se contents and temperature in Fig. 5(d), indicating a regular parabola-like behavior with temperature. Owing to the synergistic optimization of electrical and thermal properties induced by appropriate Se doping and pressure, the as-prepared BSTS-03 exhibits a maximum ZT value of 0.95 at $503 \mathrm{~K}$, which is notablely bigger than the best results of quaternary $(\mathrm{Bi}, \mathrm{Sb})_{2}(\mathrm{Te}, \mathrm{Se})_{3}$ with hole conduction obtained by theoretical calculation [15], and is very close to the maximum $\mathrm{ZT}$ value of the state-of-the-art $(\mathrm{Bi}, \mathrm{Sb})_{2}(\mathrm{Te}, \mathrm{Se})_{3}$ alloys [16]. Additionally, the thermoelectric properties of as-prepared $\mathrm{Bi}_{0.5} \mathrm{Sb}_{1.5} \mathrm{Te}_{3}$ without Se doping by HPHT method have been shown in Fig. S4. According to a comparison, the thermoelectric performances of as-prepared quaternary BSTS-03 are obviously better than that of $\mathrm{Bi}_{0.5} \mathrm{Sb}_{1.5} \mathrm{Te}_{3}$ without $\mathrm{Se}$ doping in the same synthesis condition. Therefore, the acceptable ZT value of 0.95 at $503 \mathrm{~K}$ suggests that the prospect of quaternary $(\mathrm{Bi}, \mathrm{Sb})_{2}(\mathrm{Te}, \mathrm{Se})_{3}$ is optimistic, and a further improvement in its thermoelectric properties can be expected by incorporating with the precise optimization of chemical composition, lattice/electron structure, microstructure, ultrahigh pressure, etc.

\section{Conclusions}

In summary, the quaternary $\mathrm{Bi}_{0.5} \mathrm{Sb}_{1.5} \mathrm{Te}_{3-\mathrm{x}} \mathrm{Se}_{\mathrm{x}}$ alloys with $\mathrm{x}=0.3,0.5$ and 0.75 are successfully synthesized in $25 \mathrm{~min}$ by the HPHT method. The unique triangular nanoplates are created, indicating 
that $\mathrm{Bi}_{2} \mathrm{Te}_{3}$-based compounds have the epitaxial nature according to two-dimensional triangular structures in lattices. The abundant lattice defects, which are regard as the scattering center of phonons to reduce thermal conductivity, are formed in the microstructure due to the effects of synthesis pressure and $\mathrm{Sb} / \mathrm{Se}$ co-doping. The carrier concentration is substantially modulated originating from the donor-like effect induced by defects. In addition, the Raman spectra of quaternary BSTS exhibit significant variations in Raman shift and relative intensity of $\mathrm{A}_{\mathrm{g}}{ }^{1}, \mathrm{E}_{\mathrm{g}}{ }^{2}$ and $\mathrm{A}_{\mathrm{g}}{ }^{2}$ vibration modes, suggesting the changes of crystal structures and lattice dynamics produced by $\mathrm{Se} / \mathrm{Te}$ substituents. Ultimately, with the synergistic effects of Se doping and pressure on microstructure, carrier concentration and transport behavior, an acceptable maximum ZT value of 0.95 is obtained at $503 \mathrm{~K}$ from as-prepared BSTS-03 alloys via the HPHT method.

\section{Acknowledgements}

This work was financially supported by the National Science Foundation of China (51071074 and 11464035), the Science and Technology Developing Project of Jilin Province (20140101052JC), the Open Project of State Key Laboratory of Superhard Materials (Jilin University, No. 201609) and the Open Project (Key Laboratory of Functional Materials Physics and Chemistry (Jilin Normal University), Ministry of Education, China (No. 201610)).

\section{References}

[1] J.H. Li, Q. Tan, J.F. Li, D.W. Liu, F. Li, Z.Y. Li, M.M. Zou, K. Wang, Adv. Funct. Mater. 23 (2013) 4317-4323.

[2] T.C. Harman, P.J. Taylor, M.P. Walsh, B.E. LaForge, Science 297 (2002) 2229-2232.

[3] B. Poudel, Q. Hao, Y. Ma, Y.C. Lan, A. Minnich, B. Yu, X. Yan, D.Z. Wang, A. Muto, D. Vashaee, X.Y. Chen, J.M. Liu, M.S. Dresselhaus, G. Chen, Z.F. Ren, Sicence 320 (2008) 634-638.

[4] L.P. Hu, T.J. Zhu, X.H. Liu, X.B. Zhao, Adv. Funct. Mater. 24 (2014) 5211-5218.

[5] W.J. Xie, X.F. Tang, Y.G. Yan, Q.J. Zhang, T.M. Tritt, Appl. Phys. Lett. 94 (2009) 102111.

[6] C.J. Liu, H.C. Lai, Y.L. Liu, L.R. Chen, J. Mater. Chem. 22 (2012) 4825-4831.

[7] D.H. Kim, C. Kim, S.H. Heo, H. Kim, Acta Mater. 59 (2011) 405-411.

[8] T.E. Chan, J.M. LeBeau, R. Venkatasubramanian, P. Thomas, J. Stuart, C.C. Koch, Appl. Phys. Lett. 103 (2013) 144106. 
[9] W.T. Chiu, C.L. Chen, Y.Y. Chen, Sci. Rep. 6 (2016) 23143.

[10] J.T. Im, K.T. Hartwig, J. Sharp, Acta Mater. 52 (2004) 49-55.

[11] X. Guo, X.P. Jia, K.K. Jie, H.R. Sun, Y.W. Zhang, B. Sun, H.A. Ma, CrystEngComm, 15 (2013) 7236-7242.

[12] X. Guo, J.M. Qin, X.L. Lv, L. Deng, X.P. Jia, H.A. Ma, H.S. Jia, RSC Adv. 6 (2016) 60736-60740.

[13] B. Sun, X.P. Jia, D.X. Huo, H.R. Sun, Y.W. Zhang, B.W. Liu, H.Q. Liu, L.J. Kong, H.A. Ma, J. Alloys Comp. 658 (2016) 19-22.

[14] L. Deng, L.B. Wang, X.P. Jia, H.A. Ma, J.M. Qin, Y.C. Wan, J. Alloys Comp. 602 (2014) 117-121.

[15] P. Lu, Y. Li, C. Wu, Z. Yu, H. Cao, X. Zhang, N. Cai, X. Zhong, S. Wang, J. Alloys Comp. 584 (2014) 13-18.

[16] S.Y. Wang, W.J. Xie, H. Li, X.F. Tang, J. Phys. D: Appl. Phys. 43(2010) 335404.

[17] Y.C. Lan, A.J. Minnich, G. Chen, Z.F. Ren, Adv. Funct. Mater. 20 (2010) 357-376.

[18] J. Zhang, Z.P. Peng, A. Soni, Y.Y. Zhao, Y. Xiong, B. Peng, J.B. Wang, M.S. Dresselhaus, Q.H. Xiong, Nano Lett. 11 (2011) 2407-2414.

[19] J. Sotor, G. Sohon, K. Grodecki, K.M. Abramski, Appl. Phys. Lett. 104 (2014) 251112.

[20] A. Soni, Z. Yanyuan, Y. Ligen, M.K.K. Aik, M.S. Dresselhaus, Q.H. Xiong, Nano Lett. 12 (2012) 1203-1209.

[21] K.M.F. Shahil, M.Z. Hossain, D. Teweldebrhan, A.A. Balandin, Appl. Phys. Lett. 96 (2010) 153103.

[22] T.J. Zhu, Z.J. Xu, J. He, J.J. Shen, S. Zhu, L.P. Hu, T.M. Tritt, X.B. Zhao, J. Mater. Chem. A 1 (2013) 11589-11594.

[23] L.P. Hu, H.J. Wu, T.J. Zhu, C.G. Fu, J.Q. He, P.J. Ying, X.B. Zhao, Adv. Energy Mater. 5 (2015) 1500411

[24] Z.J. Xu, H.J. Wu, T.J. Zhu, C.G. Fu, X.H. Liu, L.P. Hu, J. He, J.Q. He, X.B. Zhao, NPG Asia Materials (2016) 8, e302; doi:10.1038/am.2016.134.

[25] T.J. Zhu, L.P. Hu, X.B. Zhao, J. He, Adv. Sci. 3 (2016) 1600004.

[26] S.Y. Wang, J. Yang, T. Toll, J.H. Yang, W.Q. Zhang, X.F. Tang, Sci. Rep. 5 (2015) 10136. 


\section{Figures}
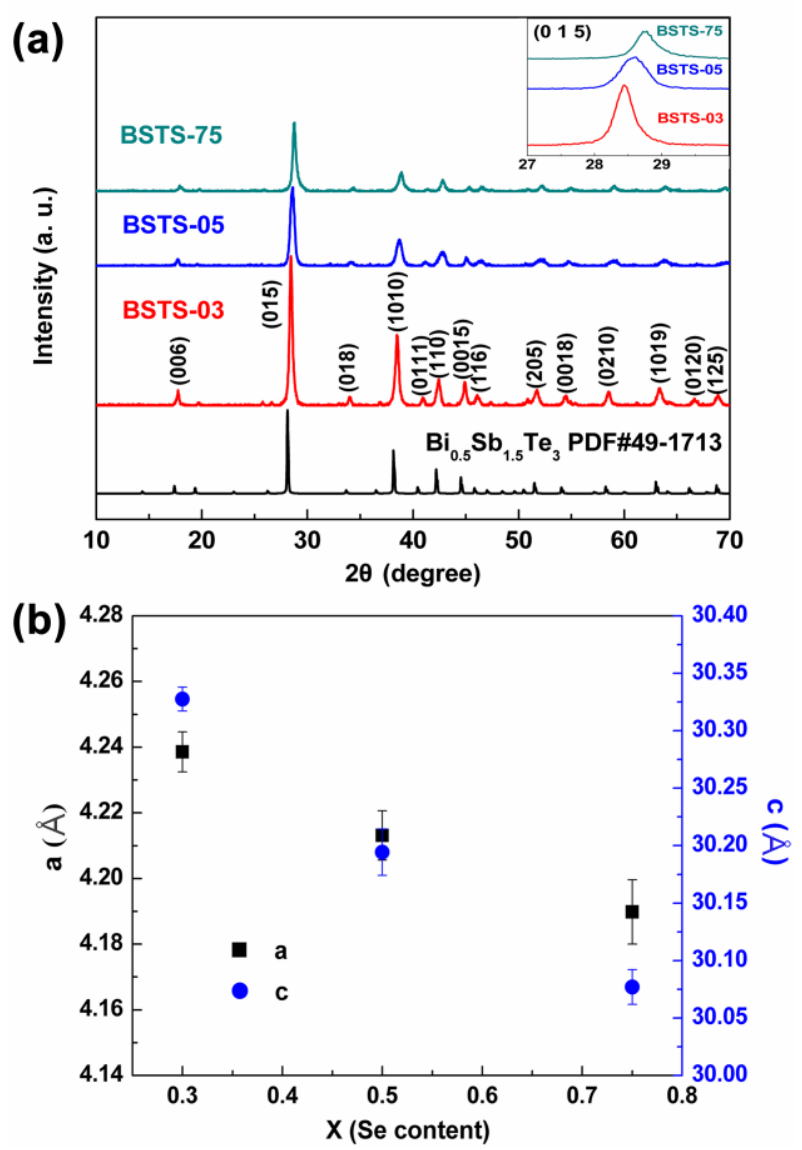

Fig. 1. (a) XRD patterns of the as-prepared BSTS with $x=0.3,0.5$ and 0.75 , the inset is the detailed view of the (015) peaks. (b) The calculated lattice parameters (a, c) of the as-prepared samples.

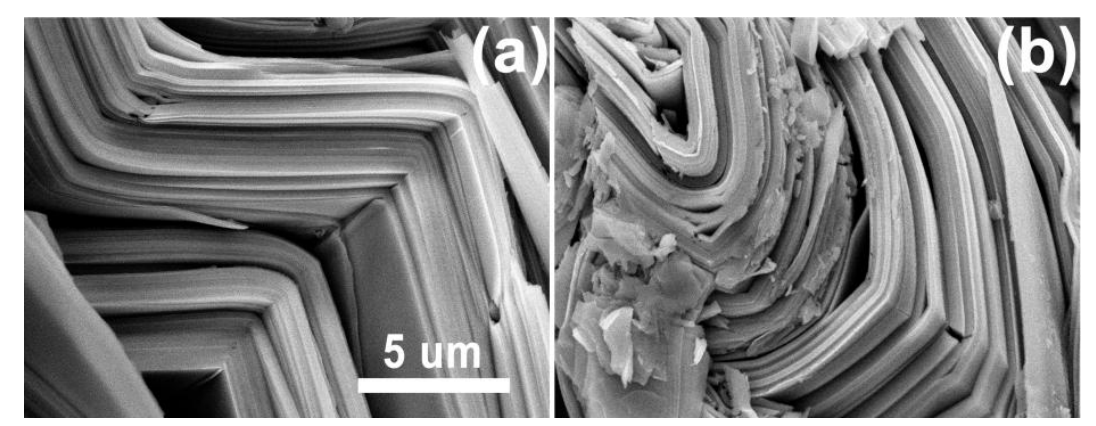

Fig. 2. FESEM micrographs of fracture surfaces of the as-prepared BSTS-03 (a) and BSTS-75 (b) alloys. 


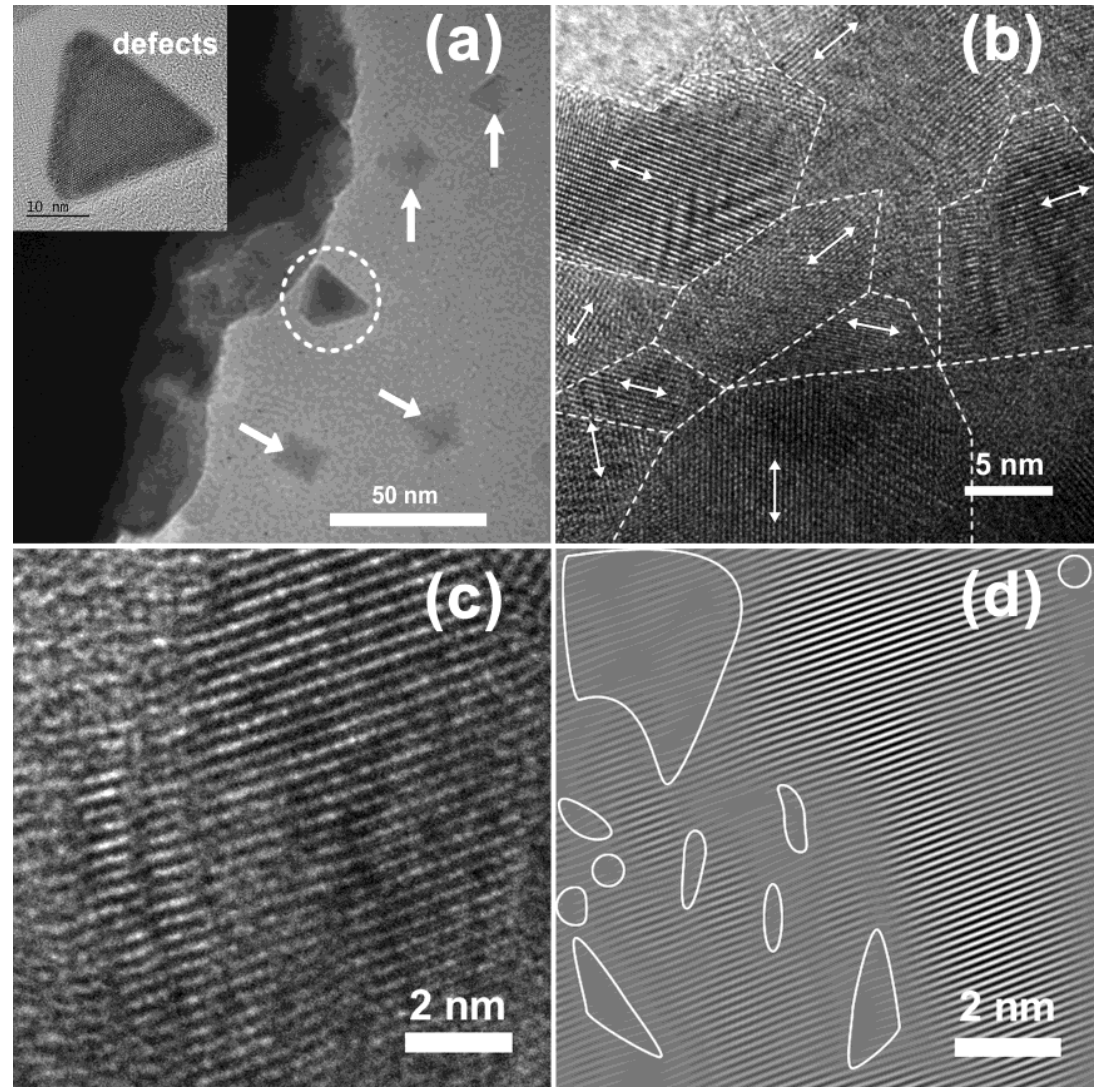

Fig. 3. (a) TEM image of the as-prepared BSTS-03, the inset is the detailed view of the triangular nanoplate in the circular region. HRTEM micrographs of the as-prepared BSTS-03. (b) Lattice orientations. (c) Lattice defects: abundant lattice distortions and dislocations. (d) IFFT image corresponding to $(\mathrm{c})$.
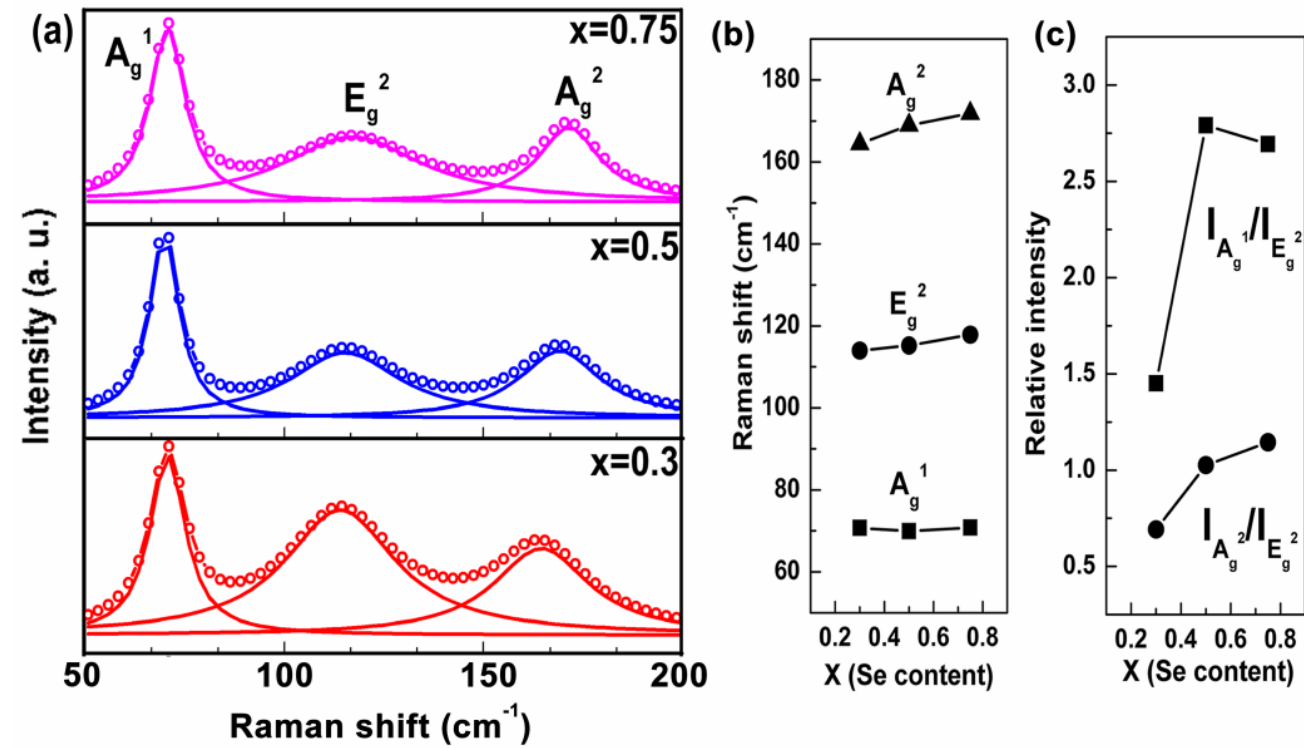

Fig. 4. (a) Raman spectra of as-synthesized $\mathrm{Bi}_{0.5} \mathrm{Sb}_{1.5} \mathrm{Te}_{3-\mathrm{x}} \mathrm{Se}_{\mathrm{x}}$ with $\mathrm{x}=0.3,0.5$ and 0.75 . The variations of three Raman active modes with different Se contents: (b) the phonon frequencies and (c) the relative intensities 

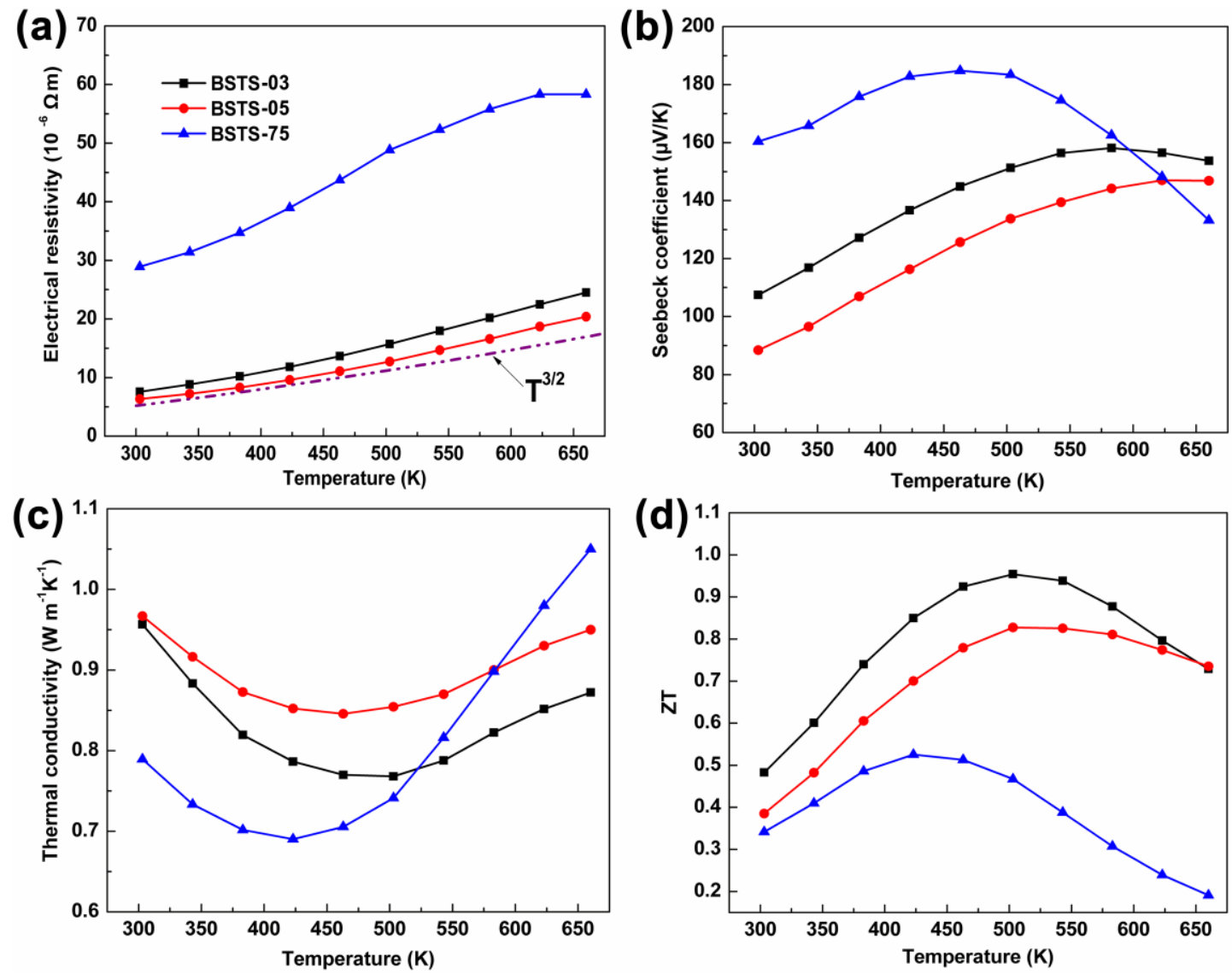

Fig. 5. Temperature dependences of the electrical resistivity (a), Seebeck coefficient (b), thermal conductivity (c) and ZT values (d) of as-prepared BSTS-03, BSTS-05 and BSTS-75 alloys. 